\title{
SUPERPOSICIÓN: LITERAL Y FENOMENAL. LA INFLUENCIA DE ANNI ALBERS EN EL GRAN ALMACÉN DE BIJENKORF DE MARCEL BREUER
}

\author{
Jon Arcaraz Puntonet
}

El artículo considera el gran almacén De Bijenkorf como un hito en la carrera de Marcel Breuer. El edificio representa un cambio desde la superposición literal a la fenomenal en lo que respecta al tratamiento de las fachadas. Esta evolución solo puede ser entendida a través de la influencia de Anni Albers en su obra. La estrategia de estratificación superficial como resultado de la cualidad de la superposición fenomenal permite al edificio adquirir su presencia característica. Un fenómeno ocurre cuando las diferentes capas se combinan en una imagen que alude a un tejido y confiere al edificio su materialidad. Entonces, solidez y transparencia se mezclan: Sol y Sombra.

Palabras clave: Yuxtaposición, Superposición, Transparencia fenomenal Keywords: Juxtaposition, Superimposition, Phenomenal Transparency

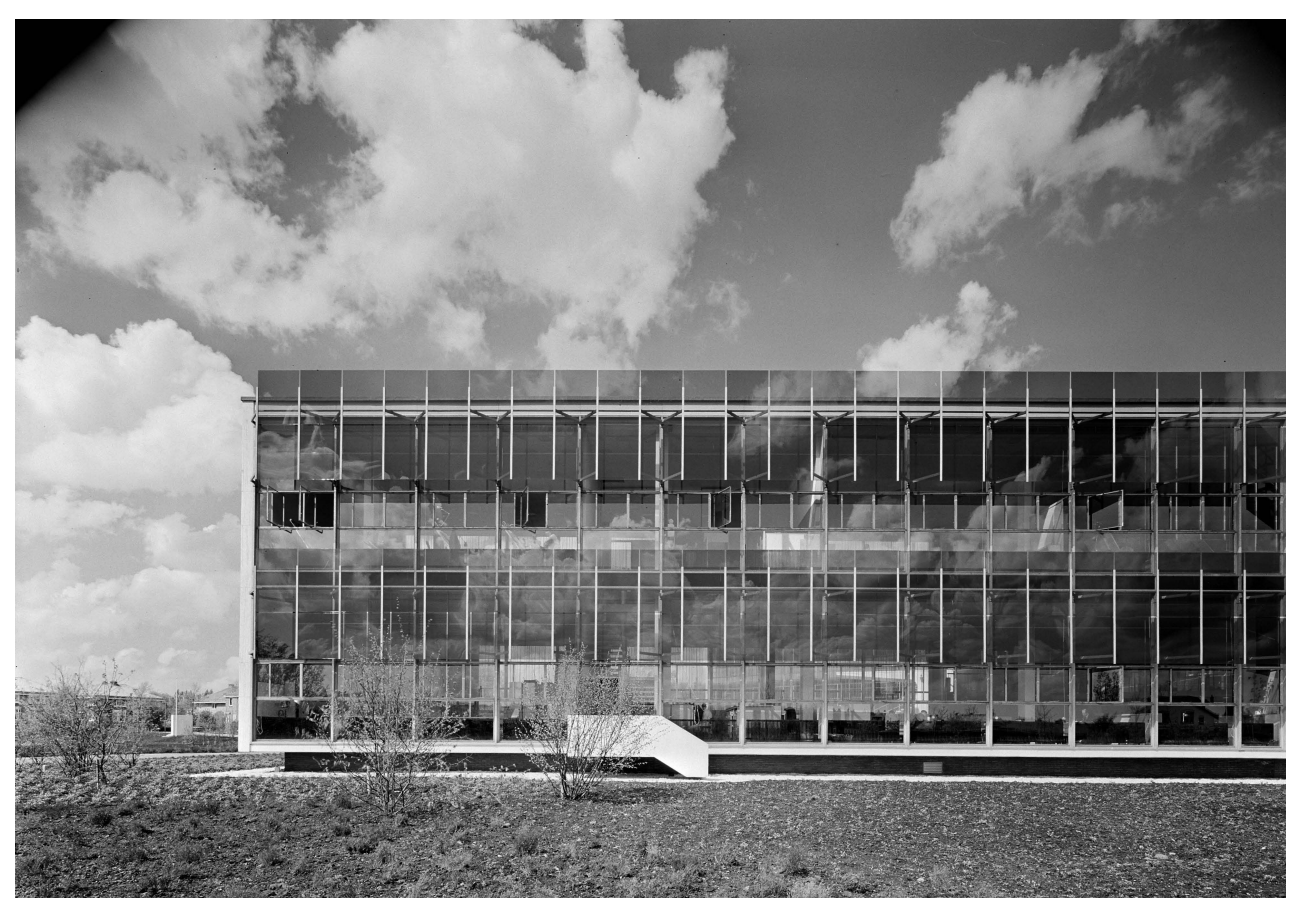

1

La tesis de este artículo es que el gran almacén De Bijenkorf (Rotterdam, 1953-1957) ilustra un paso en la evolución del trabajo de Marcel Breuer ${ }^{1}$. El cambio sólo puede ser entendido a través de los descubrimientos en superposición superficial de su colega de la Bauhaus Anni Albers. Esta estrategia llevada al campo de la arquitectura hace de las fachadas el objeto de estudio.

Con respecto a este tema, tres categorías principales pueden ser identificadas en la carrera del arquitecto: aquella iniciada en los cuarenta basada en una superposición literal de parasoles, que finalmente dio lugar a las vibraciones de los diferentes planos de vidrio utilizados; aquella iniciada en los cincuenta, en la que los proyectos holandeses construidos en piedra y basados en la noción de superposición fenomenal son determinantes; y aquella de los años sesenta y setenta en los que poco a poco la piedra dejó paso a la escala mayor del hormigón prefabricado para jugar con la cualidades esculturales del proyecto.

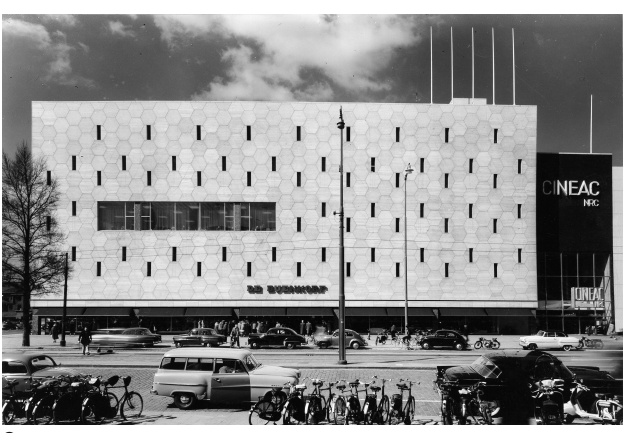

2

Fig. 1. Oficinas de la Fábrica Van Leer, Amstelveen, Holanda (1957-1958). Fotografía: Jan Versnel. Collection Maria Austria Instituut. Amsterdam

Fig. 2. Fachada Este. De Bijenkorf. Fotografía: Frits Monshouwer. Collection Het Nieuwe Instituut archive (code): ELZA inv.nr.: $77-25$

1. El edificio fue proyectado con la colaboración del arquitecto local Abraham Elzas. 


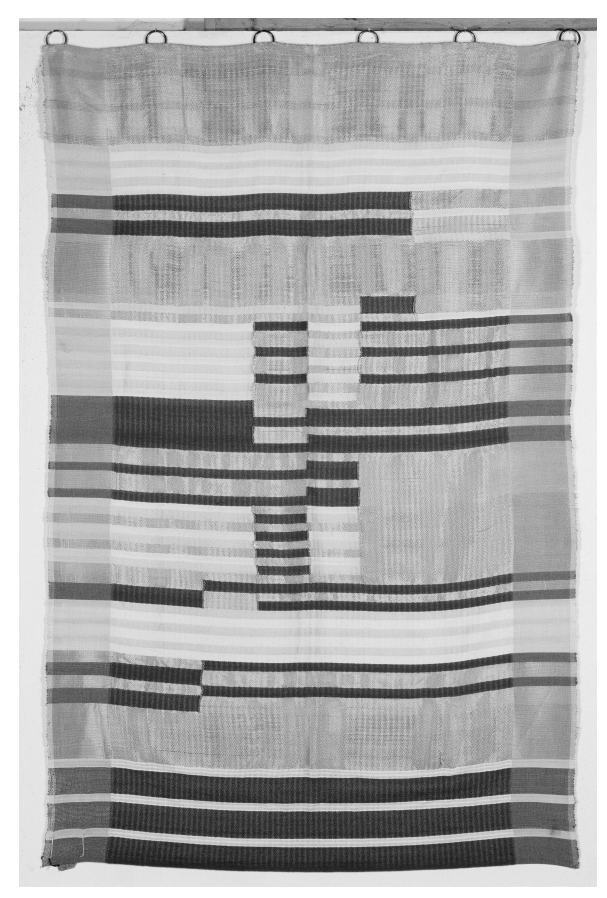

3

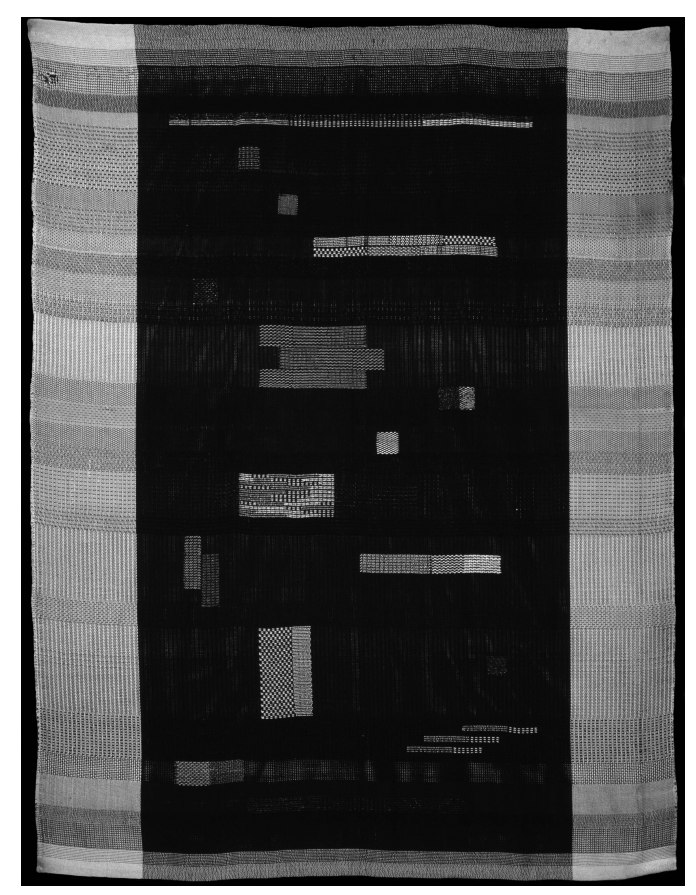

4
Fig. 3. Wallhanging, 1925. Anni Albers. silk, cotton, acetate. 50x 38 in. $(127 \times 96.5 \mathrm{~cm})$ Die Neue Sammlung, Munich 363.26 Image Courtesy of The Josef and Anni Albers Foundation.

Fig. 4. Ancient Writing, 1936. Anni Albers. rayon, linen, cotton, and jute. $59 \times 433 / 4$ in. $(149.86 \times 111.125 \mathrm{~cm}) \odot 2016$ The Josef and Anni Albers Foundation / Artists Rights Society, New York. Collection Smithsonian American Art Museum, Washington D.C. Image Courtesy of The Josef and Anni Albers Foundation (code): 1936.12.2

2. BREUER Marcel, Sun and Shadow: The Philosophy of an architect, Dodd, Mead \& Company, New York, 1995, p. 34.

3. Poco después, en el invierno de ese mismo año, Colin Rowe y Robert Slutzky escribirían Transparencia: literal y fenomenal (parte 1 y 2). Los Texas Rangers explicitaron sus conceptos recurriendo también a la Teoría de la Gestalt. Sin embargo, su aproximación desde el collage les conducía a otros intereses diferentes a aquellos de los miembros de la Bauhaus. Esta es la razón por la que este artículo se refiere a la transparencia como superposición.

4. BREUER, Marcel, What is happening to Modern Architecture? Museum of Modern Art, New York, 1948.

5. Sin embargo, su interés por los textiles americanos se originó en su juventud. Como explica Virgina Gardner en su tesis doctoral de 1997 Anni Albers: The Significance of Ancient American Art For Her Woven And Pedagogical Work esta referencia ya estaba presente en la vida alemana anterior a la Primera Guerra Mundial en el Berlin Museum fur Volkerkunde que adquirió textiles andinos debido a sus trabajos arqueológicos, también lo estaba en el discurso primitivista en Alemania.
Sin embargo, este artículo se centra exclusivamente en el cambio de la primera a la segunda categoría.

El paradigma de los cuarenta fue influenciado por los estudios climáticos de los hermanos Olgyay. El parasol fue uno de los tipos que Breuer proyectó para controlar el confort interior del edificio. El punto álgido de esta superposición literal puede ejemplificarse con la Sede para la Organización Educacional, Científica y Cultural de los Estados Unidos (U.N.E.S.C.O.) en París, aunque un ejemplo más extremo podría ser las Oficinas de la Fábrica Van Leer. En ambos casos, la fachada de vidrio con un parasol vertical superpuesto, también de vidrio, tenía como resultado una superposición literal finalmente cualificada por su transparencia (Fig. 1).

Sin embargo, en los cincuenta, Breuer ensayó otras soluciones además de las ya experimentadas (Fig. 2). Sus intereses quedaron planteados en el libro Sol y Sombra:

\footnotetext{
"La transparencia es indudablemente uno de nuestros objetivos. Es una de las más fascinantes nuevas posibilidades tecnológicas. Podemos conseguirla con los medios que tenemos ... pero la transparencia necesita también de la solidez. Y no sólo por razones estéticas - sino también porque la transparencia total deja de lado tales consideraciones como la privacidad, las superficies reflectantes, la transición del desorden al orden, el amueblamiento, un fondo para ti, para tu vida cotidiana. La transparencia gana al lado de la solidez - y la solidez hace que funcione. Sol y Sombra"².
}

Marcel Breuer se estaba refiriendo a contrastes entendidos como correspondencias binarias $^{3}$. Esta era una idea que ya había planteado en las décadas anteriores. Su adopción del termino 'Sol y Sombra'4 fue simplemente un modo de expresar y expandir el ámbito de su teoría.

Esta cita un tanto críptica venía acompañada de la exposición de sus proyectos. Por ello, conviene centrarse en su obra construida para intentar desentrañar los tipos a los que recurrió para desarrollar su pensamiento. En este sentido, el gran almacén De Bijenkorf supuso la adopción de una nueva estrategia: comenzó una investigación en superposición superficial influenciado por el trabajo de Anni Albers que caracterizaría sus diseños posteriores.

La influencia de Anni Albers tiene que ver con su evolución desde 1936 en adelante a raíz de su primer viaje a Méjico para conocer de primera mano los textiles mayas ${ }^{5}$. Este aprendizaje supuso el paso de un diseño basado en la estructura del tejido a otro más espontáneo en el que motivos figurativos eran superpuestos sobre el fondo estructural (Figs. 3 y 4 ). 


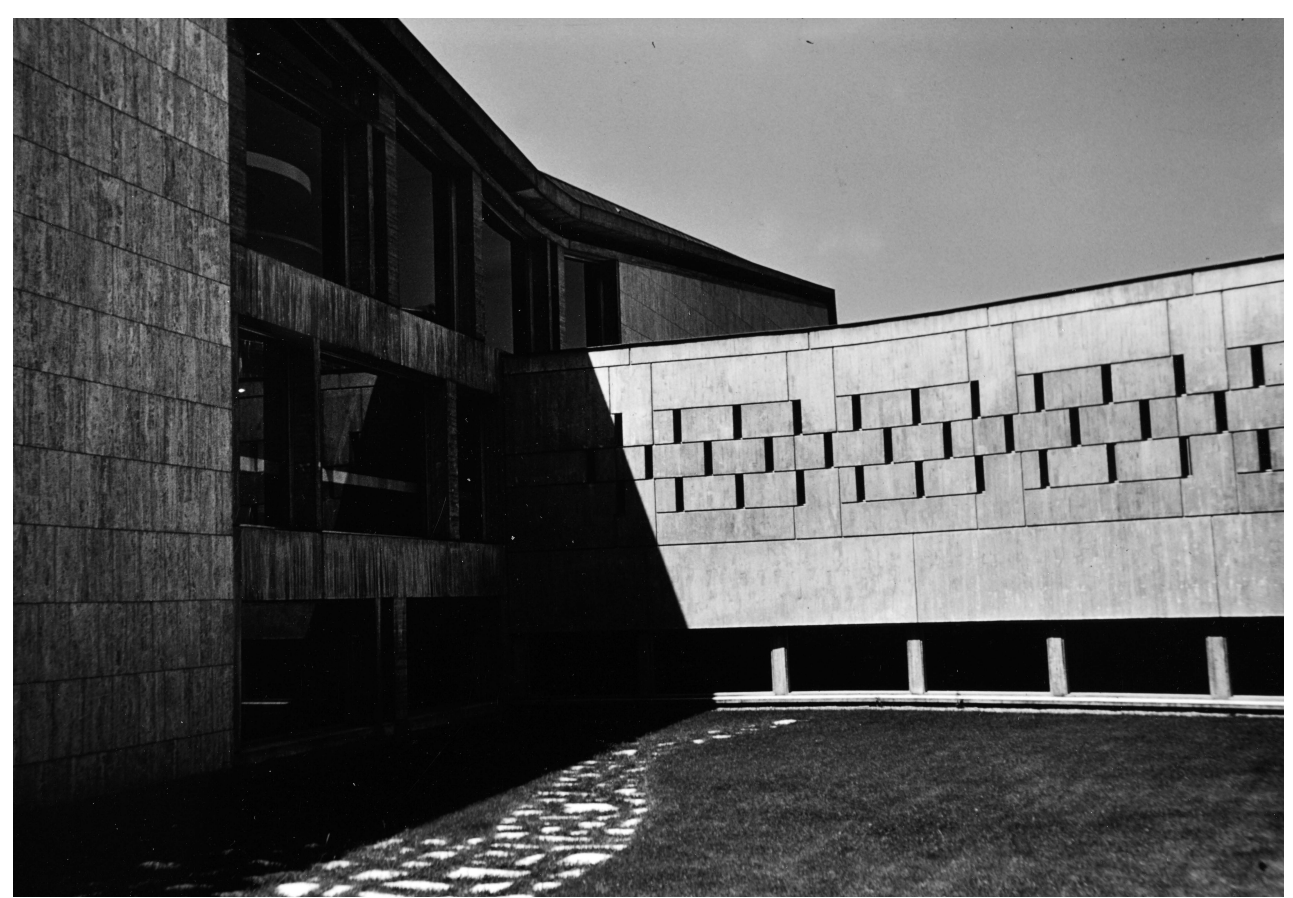

5

"Anni Albers persiste y perfecciona el ejercicio hasta hacer de la cuadrícula un soporte visual, que sostiene y posibilita las diferentes variaciones modulares de las relaciones entre fondo y figura"

El progreso de Anni Albers inspiró a Breuer en su proyecto holandés. Comparando uno de los muros de la U.N.E.S.C.O. con aquel de De Bijenkorf se pueden observar ciertas diferencias. El primero fue construido separando las unidades de piedra para dejar huecos entre ellas de tal manera que las fuerzas gravitacionales se hicieran evidentes. Mientras, la fachada del gran almacén tenía una capa de huecos superpuesta a la trama de piedra. Se trataba de un nuevo patrón que se superponía a una cuadrícula consolidada (Figs. 5 y 6).

Por ello, se puede afirmar que fue una aproximación textil la que movió a Breuer en su proyecto del gran almacén. Su manera de pensar sería la misma que la de Anni Albers. Un textil esta hecho de un tejido con un tipo de fibra específico. Ambos la fibra y el tejido cualificaban la textura del producto final. Además, un patrón puede ser superpuesto a la estructura anterior. Aunque este último es manufacturado cosiendo fibras de diferente color, el resultado es que el patrón es una capa superpuesta a aquella del tejido. Entonces, la textura y el patrón se solapan y pueden ser tratados como formas diferentes. El uso de contrastes y armonías de formas (patrones) y superficies (texturas) estaban en el origen de este diseño. Un análisis de estas relaciones contribuirá a aclarar la descripción del edificio.

En el caso de De Bijenkorf, la elección del material de la fachada influía en muchas características del proyecto y ésta es la razón por la que Breuer partió de esta decisión esencial. El material elegido, la piedra, tenía varias peculiaridades. Su manufactura en losas permitía diseñar una fachada ventilada como sistema constructivo. Esa fachada estaría libre de las restricciones de la estructura portante. El plano de fachada podría entonces ser utilizado como un material de cubrición. Por lo tanto, la idea de tejido ya estaba implícita en la elección de una fachada ventilada. Sin embargo, el proyecto desarrolló otras estrategias que reforzarían su percepción.

Los despieces de cada lienzo de fachada adquirieron diferentes formas. La no direccionalidad del patrón hexagonal tenía como propósito la percepción del lienzo como una trama unitaria en la que la gravedad pasaba a un segundo plano. Esta misma idea fue también perseguida en el otro patrón rectangular: la disposición a mata-junta con unas losas de proporción vertical buscaba contrarrestar la apariencia de peso (Fig. 7). Con todo, la relación entre las dos puede también ser comparada con algunas de las construcciones te-

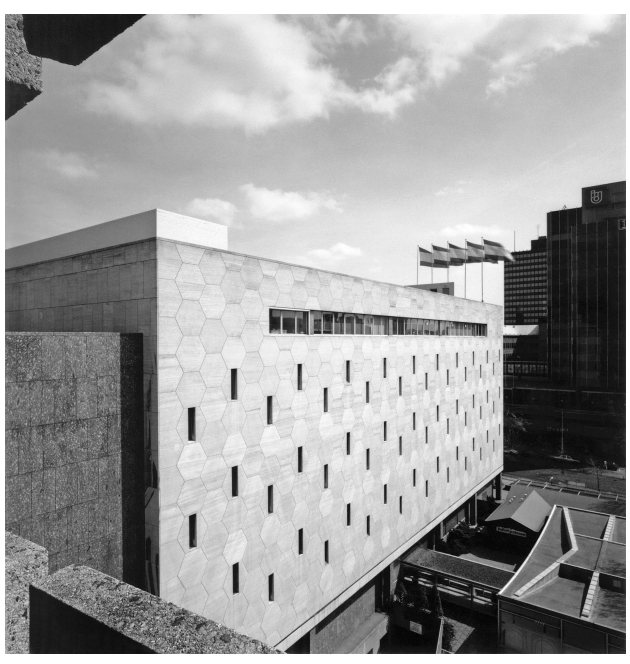

6

Fig. 5. Fachada. Sede de la U.N.E.S.C.0., Paris (1952-1958). Collection Marcel Breuer Papers, Box 12, Folder 67, Archives of American Art, Smithsonian Institution, Washington D.C.

Fig. 6. Fachada Oeste. De Bijenkorf. Fotografía: Anja de Jong. (C) Anja de Jong. Collection Het Nieuwe Instituut archive. (code): ELZA inv.nr.: box ELZA 0029
6. W.AA., ALBERS JOSEF, Anni, Viajes Por Latinoamérica, Museo Nacional Centro de Arte Reina Sofía, Madrid, 2006, p. 46. 


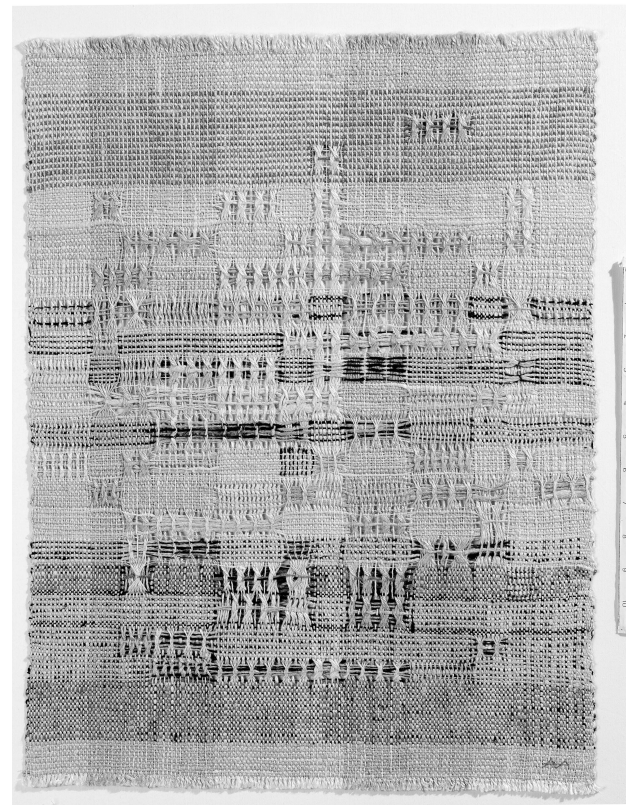

8

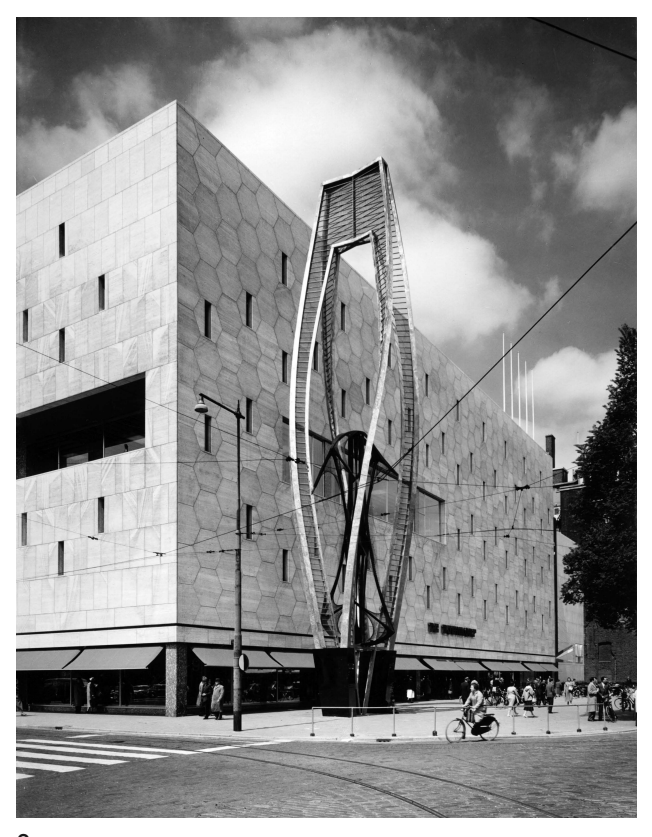

9

Fig. 7. Alzados. De Bijenkorf. Syracuse Univ. Archive.

Fig. 8. Development in Rose I, 1952. Anni Albers. linen, plain and gauze weave pictorial weaving. $221 / 2 \times 171 / 4$ in. (57.15 $x 43.815 \mathrm{~cm}$ ) (c) 2016 The Josef and Anni Albers Foundation / Artists Rights Society, New York. (code): 1996.12.2.

Fig. 9. Detalle de esquina. De Bijenkorf.

Fotografía: Spies, Amsterdam. Collection Het Nieuwe Instituut archive (code): ELZA inv.nr.: d288.

7. Ver otros ejemplos de yuxtaposición de texturas de Ann Albers en LECLERCQ, Jean-Paul, On the Structure of the Weavings en WEBER, Nicholas Fox, TABATABAI Pandora, Anni Albers, Guggenheim Museum, New York, 1999-2000.

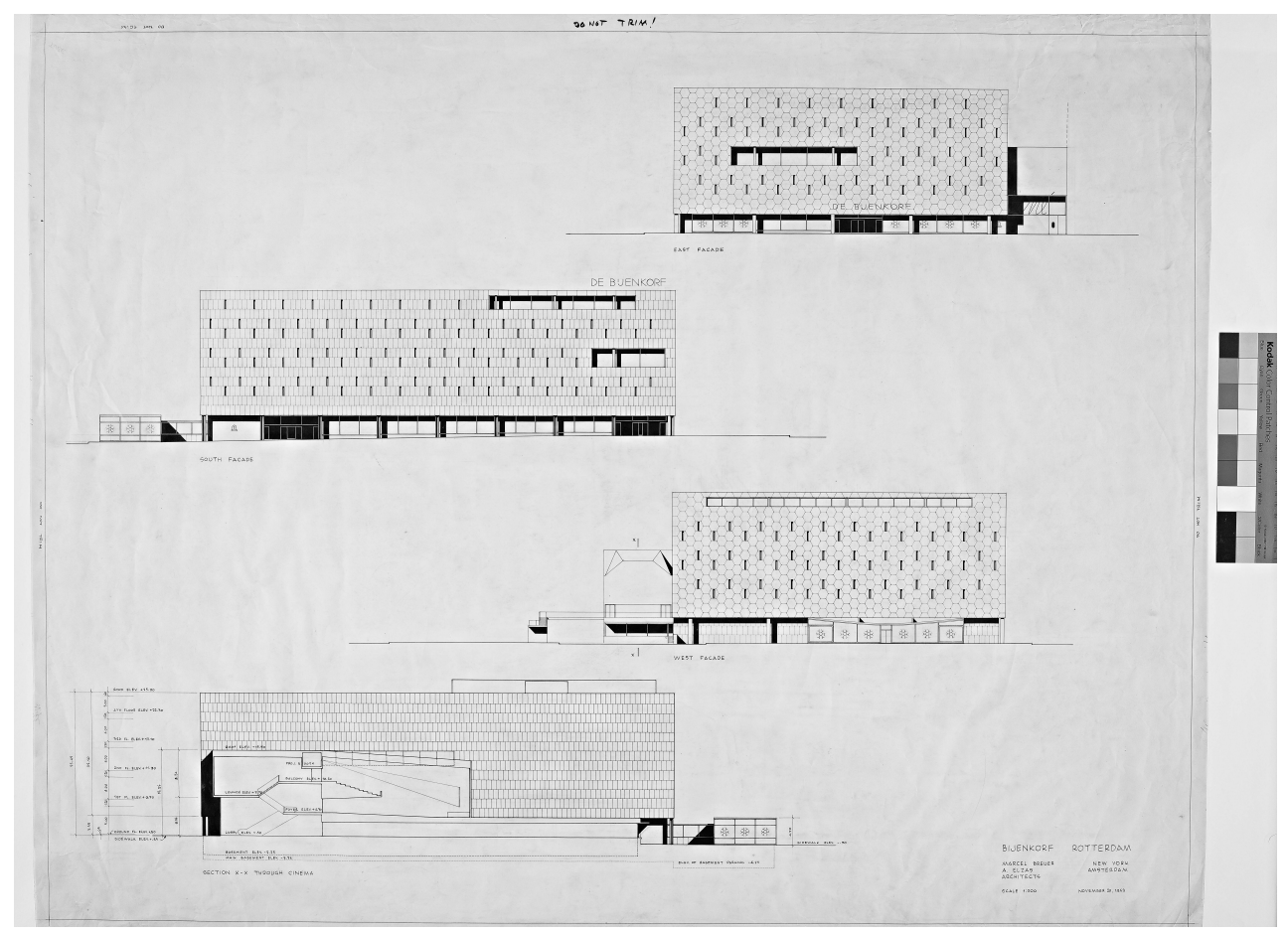

7

jidas de Anni Albers que jugaban con diferentes texturas ${ }^{7}$. Era como si dos construcciones tejidas fueran yuxtapuestas, una al lado de la otra, complementándose, cada una con su propia textura (Figs. 8 y 9).

Además de la mencionada hasta ahora, otra textura también fue utilizada. El despiece representaba una tela tejida mientras que la textura de cada losa recordaba a las fibras. Las losas de travertino fueron cinceladas de tal manera que tenían hendiduras sesgadas. La acción del polvo y la lluvia en la alternante secuencia de rayas recordaría desde lejos las arrugas de cualquier tejido secándose (Fig. 10).

Otra característica de este tejido sería aquella de su patrón de ventanas aspilleras. Aunque estaba horadado en la superficie de fachada, podría entenderse como una capa superpuesta que unificaba los diferentes lienzos. La razón funcional de estas ventanas aspilleras era la de permitir a los consumidores ver los colores de las telas en venta con la luz del día. Sin embargo, no hay lugar a duda de que Breuer estaba jugando con este otro significado de un patrón de rectángulos, dispuestos a intervalos equidistantes en el paramento exterior, superpuesto a la estructura del tejido y completamente independiente de otro tipo de ventanas grandes que tenían otros propósitos.

Cuando este tejido se doblaba en la esquina, aparecía otro detalle importante del diseño (Fig. 9). Aunque el despiece de cada fachada era distinto y el detalle constructivo de la esquina estaba resuelto con el canto visto de uno de los planos revestidos de fachada, existían otros elementos que reforzaban la continuidad. Por un lado, el patrón de ventanas aspilleras $\mathrm{y}$, por otro, la continuidad en altura y forma general de las grandes ventanas del restaurante creaban la percepción de continuidad entre diferentes planos. Esto era aun mas claro de noche cuando estos caracteres, iluminados con luz artificial, prevalecían.

La idea de unas cortinas de teatro sólidas también fue reforzada por otras dos cualidades: la ingravidez de la caja del volumen principal del edificio y el posicionamiento y tipo de algunas ventanas abiertas en ese tejido. Primero, la caja era percibida flotando en el aire como resultado del retranqueo de la planta baja. Segundo, las largas aberturas horizontales de la fachada, con carpinterías enrasadas en el trasdós de las losas de piedra, estaban situadas en una posición alta o media en los planos de fachada para crear la sensación de ligereza. 


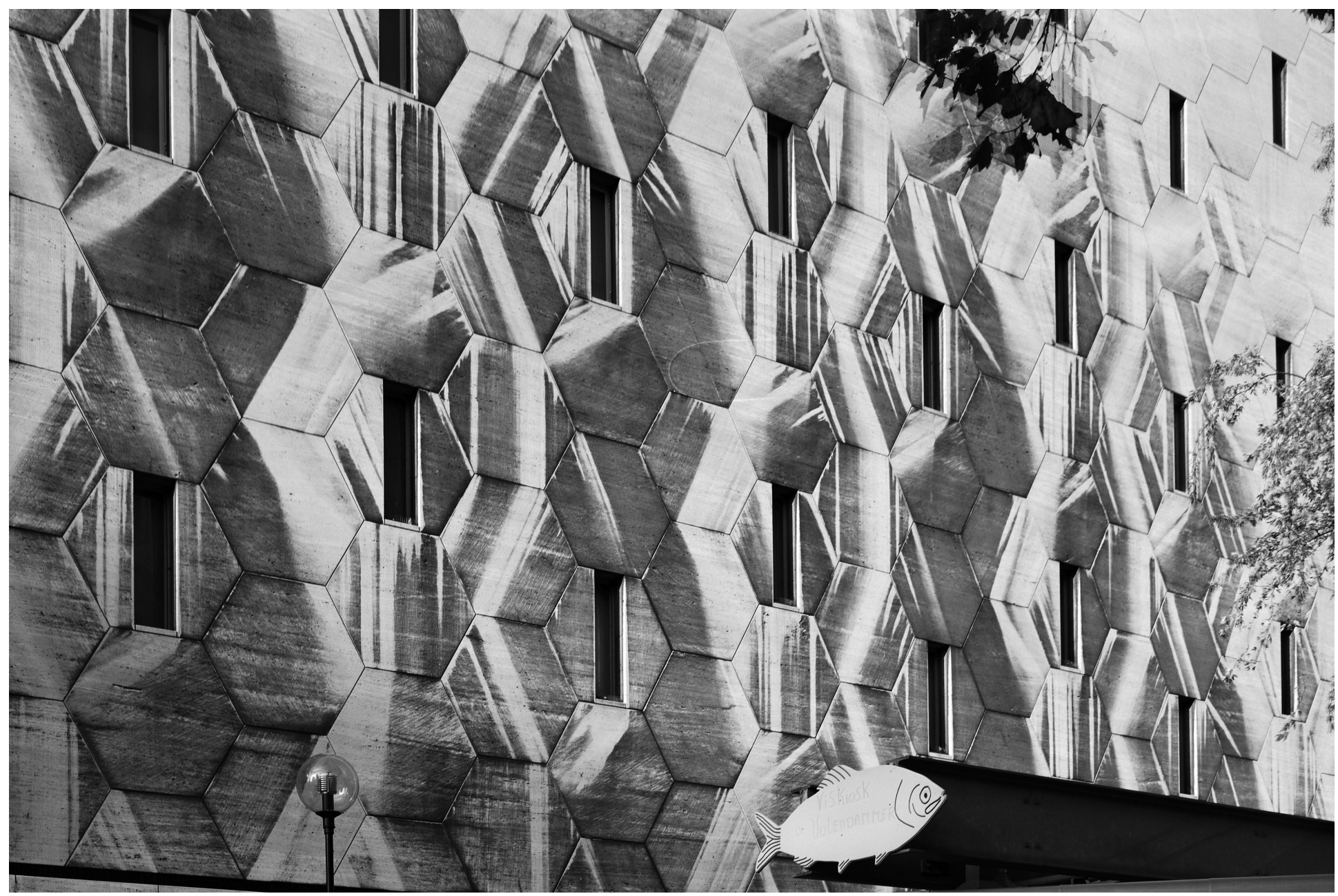

En este sentido, existían unas relaciones comparativas de profundidad entre los diferentes tipos de huecos de fachada. Así, las ventanas aspilleras parecían elementos superficiales en comparación con las grandes aberturas profundas.

Estas últimas jugaban con lo volumétrico, pero todas ellas permitían a propósito el paso de la estructura vertical, que estaba revestida con granito portugués verde oscuro, a través de los huecos de las grandes aberturas. Era la evidencia de que el edificio estaba estratificado en diferentes planos.

En primer lugar, el volumen principal fue establecido como un fondo en términos gestálticos. Las aberturas largas y profundas del restaurante recalcaban el tratamiento volumétrico. En segundo lugar, los planos de fachada que formaban parte de ese volumen eran también abordados en términos superficiales, no como la superficie del volumen en sí sino como un tejido superpuesto a él. A su vez, este tejido tenía diferentes capas que lo componían. Estas deben ser entendidas como transparentes y superpuestas. Esas capas eran aquella del patrón de ventanas aspilleras y aquella de la textura de losas de travertino. Este último patrón variaba de una fachada a otra debido a las diferentes formas de las losas de piedra. Esto permitía al arquitecto jugar con distintas vibraciones que serían percibidas desde diferentes distancias (Fig. 11).

Esta sensualidad táctil de las cualidades físicas del material fue abordada por Anni Albers en los términos que siguen:

"Si hoy en día, nos embarcáramos en la tarea de elegir telas guiados por una mente libre antes de quedar absorbidos en los placeres espontáneos que el color, la superficie, y el tacto del tejido nos dan, nuestras habitaciones parecerían ordenadas, espaciosas y serenas. Estarían animadas por esas cualidades de los ma-
Fig. 10. Textura de la fachada. De Bijenkorf. 2004-2005. Fotografía: Jannes Linders. 
Fig. 11. Vista General. De Bijenkorf.

Fotografía: Frits Monshouwer. Collection Het Nieuwe Instituut archive (code): ELZA inv.nr.: f7-22.
8. ALBERS, Annie, "The Pliable Plane: textiles in Architecture", en Perspecta The Yale Architectural Journal, 4, 1957.

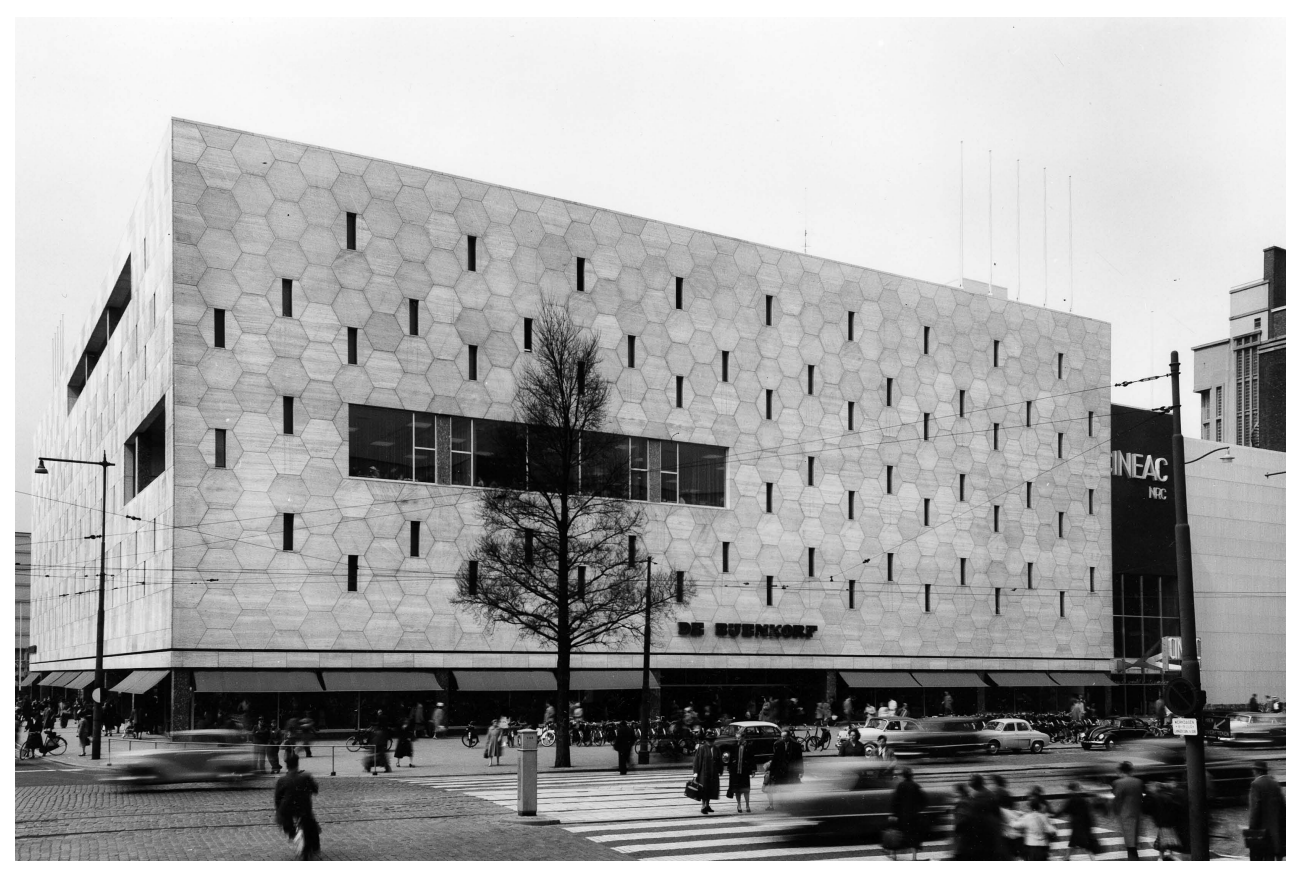

teriales que conocemos tan íntimamente por el hecho de llevarlos puestos: por el uso cerca de nuestra piel. Y si pensamos en la ropa como una segunda piel deberíamos trasladar este pensamiento y darnos cuenta de que la envolvente de las paredes es, de alguna manera, una tercera cubrición, que nuestra habitación es otro "hábito".

En conclusión, fue la estrategia de estratificación superficial como resultado de la cualidad de la superposición fenomenal la que permite al edificio adquirir su presencia característica. Un fenómeno ocurre cuando las diferentes capas se combinan en una imagen que alude a un tejido y confiere al edificio su materialidad. Entonces, solidez y transparencia se mezclan: Sol y Sombra. Como resultado de esta nueva estrategia, este proyecto de De Bijenkorf puede ser considerado como un hito en la evolución de Marcel Breuer. 